\title{
A favorable outcome of advanced dermatofibrosarcoma protuberans under treatment with sunitinib after imatinib failure
}

This article was published in the following Dove Press journal:

OncoTargets and Therapy

\author{
Wei Xiao ${ }^{1,2}$ \\ Yi Que ${ }^{1,2}$ \\ Ruiqing Peng ${ }^{1,2}$ \\ Ya Ding ${ }^{1,2}$ \\ Jingjing Zhaol,2 \\ Xizhi Wen ${ }^{1,2}$ \\ Desheng Weng ${ }^{1,2}$ \\ Xiaoshi Zhang ${ }^{1,2}$ \\ Yuanxiang Guan ${ }^{2,3, *}$ \\ Xing Zhang ${ }^{1,2, *}$ \\ 'Melanoma and Sarcoma Medical \\ Oncology Unit, Sun Yat-sen University \\ Cancer Center, Guangzhou, People's \\ Republic of China; ${ }^{2}$ State Key \\ Laboratory of Oncology in South \\ China, Collaborative Innovation \\ Center for Cancer Medicine, Sun \\ Yat-sen University Cancer Center, \\ Guangzhou, People's Republic of \\ China; ${ }^{3}$ Department of Gastric \\ Surgery, Sun Yat-sen University \\ Cancer Center, Guangzhou, \\ People's Republic of China \\ *These authors equally contributed \\ to the work
}

\begin{abstract}
While traditional cytotoxic agents play a limited role in advanced dermatofibrosarcoma protuberans (DFSP), the treatment of sunitinib for patients with advanced DFSP after imatinib failure is not well defined. The objective of this case report was to analyze the relationship between molecular mechanisms and clinical outcomes of sunitinib treatment in patients with advanced DFSP after imatinib failure. In this case report, a 37-year-old man suffered from advanced DFSP progression after surgical operation, microwave ablation, and chemotherapy. The immunohistochemistry in this patient revealed abundant expression of platelet-derived growth factor receptor-beta on tumor cells, which is one of the drug targets of sunitinib. The nucleotide sequence analysis revealed COL1A1-PDGFB fusion transcripts in this patient. Thus, we treated the patient with sunitinib, a multi-targeted tyrosine kinase inhibitor, after imatinib failure. After treatment with sunitinib, the patient exhibited a partial response and 9 months' progressionfree survival without significant adverse drug effects. In our case, the patient with advanced DFSP experienced a favorable outcome in 9-months' progression-free survival and a significant improvement of quality of life without serious side effects after sunitinib treatment. Therefore, sunitinib could serve as another treatment option for patients with advanced DFSP.
\end{abstract}

Keywords: COL1A1-PDGFB fusion gene, dermatofibrosarcoma protuberans, platelet-derived growth factor receptor-beta, sunitinib

\section{Introduction}

Dermatofibrosarcoma protuberans (DFSP) is a rare mesenchymal tumor with intermediate grade malignancy, which is infrequently capable of transferring into high-grade fibrosarcomas. It usually appears in adults, and is frequently located on the trunk, groin, lower extremity, and seldom in the head and neck. Local surgical resection with wide margins is still an accepted treatment. ${ }^{1}$ However, DFSP has a high tendency for local recurrence and a small risk of distant metastasis after excision. ${ }^{2}$ The fusion between the collagen type I alpha 1 gene (COL1A1) and the platelet-derived growth factor (PDGF)-chain gene (PDGFB), which are located in 17q22 and 22q13, respectively, is the characteristic of DFSP. ${ }^{3}$ The expression of COL1A1-PDGFB fusion protein leads to overexpression of PDGF and promotes continuous activation of PDGFR-beta in DFSP cells. ${ }^{4}$

Sunitinib is a small inhibitor of multi-tyrosine protein kinases such as plateletderived growth factor receptor (PDGF-R), vascular endothelial growth factor receptor, stem cell factor receptor, fetal liver tyrosine kinase receptor, and colony-stimulating factor receptor. It blocks multiple intracellular signaling pathways, reduces tumor vascularization and triggers cancer cell apoptosis, which results in tumor shrinkage.
Correspondence: Xing Zhang Melanoma and Sarcoma Medical Oncology Unit, Sun Yat-sen University Cancer Center, Guangzhou 510060, People's Republic of China

Tel +862087343629

Email zhangxing@sysucc.org.cn 
The range of application for sunitinib comprises gastrointestinal stromal tumors (GIST), neuroendocrine tumors, and advanced renal cell cancer. ${ }^{5}$

Herein, we have presented the case of a 37-year-old man who suffered from a progression of advanced DFSP after surgical operation, microwave ablation, and chemotherapy. We detected the COL1A1-PDGFB fusion transcripts and over-expression of PDGFR-beta in this patient so that we treated the patient with sunitinib after imatinib failure, whose targets include PDGFR-beta in developing the process of DFSP. Eventually, the patient experienced a favorable outcome after sunitinib treatment.

\section{Case report}

In December 2011, a 37-year old, previously healthy man found a painless mass on his left partes temporalis. In January 2012, the patient underwent radical functional dissection and histopathological examination showed DFSP. The postoperative computed tomography imaging technique (CT) in February 2012 (1 month after initial diagnosis) revealed multiple nodular metastases in both lungs. The patient was then treated with doxorubicin (ADM, $\left.50 \mathrm{mg} / \mathrm{m}^{2}\right)$, ifosfamide (IFO, $7.5 \mathrm{~g} / \mathrm{m}^{2}$ ) and dacarbazine (DTIC, $1 \mathrm{~g} / \mathrm{m}^{2}$ ) (3 weeks for one course) followed by a therapeutic evaluation of progressive disease (PD). Subsequently, the patient received a combined therapy with lung metastatic tumor microwave ablation and a chemotherapy regimen of gemcitabine (GEM, $1 \mathrm{~g} / \mathrm{m}^{2}$ ) and paclitaxel liposome $\left(90 \mathrm{mg} / \mathrm{m}^{2}\right)$ (3 weeks for one course) in May 2012. In September 2012, on account of the recurrence of two masses on the left side of the lumbar part and right side of the abdominal wall ( 8 months after initial diagnosis), the patient underwent palliative tumor resection on the abdominal wall. The histopathological examination revealed DFSP.

Owing to multiple systemic metastases, poor general condition and suboptimal compliance, the patient indicated that he could not tolerate standard treatment including chemotherapy and radiation therapy anymore. As the PDGF signaling pathway was described to be involved in the development of DFSP, we detected the molecular profile of the tumor with informed consent from the patient. The paraffin-embedded tissue sections of this patient were stained with a rabbit polyclonal anti-PDGFR-beta antibody (1:400, Santa Cruz Biotechnology Inc., Dallas, TX, USA, sc-339) by immunohistochemistry. We also detected the COL1A1-PDGFB fusion transcripts by reverse transcription polymerase chain reaction and the PCR primer sequences, which were previously described by Patel et al and which are presented in Table $1 .^{6}$
Table I Primer sequences of the COLIAI and PDGFB gene

\begin{tabular}{ll}
\hline Primer & Nucleotide sequence $\left(\mathbf{5}^{\prime} \rightarrow \mathbf{3}^{\prime}\right)$ \\
\hline COLIAI (forward) & \\
Exon 5 & GCATCC CTG GAC AGC CTG GA \\
Exon 8 & AAG GCT TCC AAG GTC CCC CT \\
Exon 12 & GTG CCA AGG GAG ATG CTG GT \\
Exon I7 & GTG CTG TTG GTG CTA AGG GTG A \\
Exon 20 & AGG AGA CAC TGG TGC TAA GGG AGA \\
Exon 23 & CTG GTC TGC CTG GTG CCA A \\
Exon 27 & TGC TGG CAA AGATGG AGA GGC T \\
Exon 31 & CGA GAG AGG TTT CCC TGG CGA \\
Exon 32 & TGA ACG TGG TGC AGC TGG TCT TC \\
Exon 36 & GCG AAC CTG GTG ATG CTG GT \\
Exon 39 & GGC AAA GAA GGC GGC AAA GGT \\
Exon 40 & GGA GAG AGA GGC TTC CCT GGT CTT \\
Exon 43 & CCT GCT GGC AAG AGT GGT GAT \\
Exon 46 & CTG GTG AAC AAG GTC CCT CTG GA \\
Exon 48 & CCA CCT CAA GAG AAG GCT CAC GA \\
Exon 49 & GCA ACC TGG ATG CCATCA AAG TCT T \\
Exon 50 & GCC TCC CAG AAC ATC ACC TAC CA \\
Exon 5I & GCA AGA CAG TGATTG AAT ACA AAA CCA \\
PDGFB (reverse) & \\
Exon 2 & ATC AAA GGA GCG GAT CGA GTG GTC \\
\hline
\end{tabular}

Note: Data from Patel et al. ${ }^{6}$

Abbreviation: PDGFB, platelet-derived growth factor-chain gene.

The experimental results showed the COL1A1-PDGFB fusion transcripts and over-expression of PDGFR-beta in paraffin-embedded samples of this patient (Figure 1). The results suggested that the PDGFR-targeted agents might be an appropriate treatment for this tumor. Therefore, in the first month of 2013, the patient initiated oral therapy with imatinib and experienced stable disease during the regular repeated examinations. However, an abdominal CT scan with contrast enhancement showed tumor growth in the lung and a new metastasis in the pancreas in May 2014 (28 months after initial diagnosis) and the general condition of the patient showed significant deterioration.

As we know, sunitinib inhibits cellular signaling by targeting multiple receptor tyrosine kinases including PDGFR. So, in the absence of a proven effective systemic therapy for this case (the first reliable report on DFSP in sunitinib treatment was in May 2015), we decided to treat our patient with sunitinib in October 2014. ${ }^{7}$ The dosing schedule was $37.5 \mathrm{mg}$ once daily, taken orally for 4 consecutive weeks, followed by a 2 -week rest period (schedule $4 / 2$ ). The patient tolerated this dosage well and the adverse reaction was mild, except for grade 2 hypertension, which subsided under the treatment of Amlodipine. The tumor size on the left upper arm was shrinking and a regular CT/MRI scan showed partial response in the lung and pancreas metastases over 2 months from initiation of sunitinib (35 months after initial diagnosis) (Figure 2). Therefore, our patient continued the sunitinib therapy and during this treatment, he experienced stable 
A

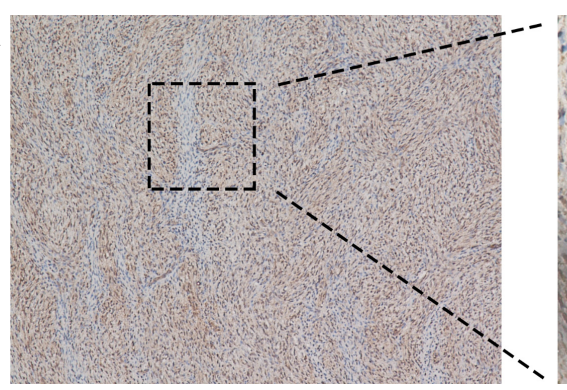

$100 \times$

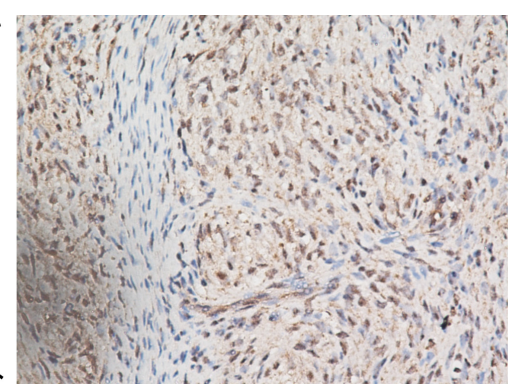

$400 \times$

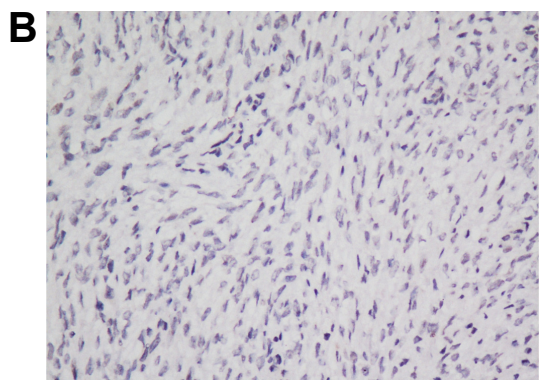

$400 \times$
C

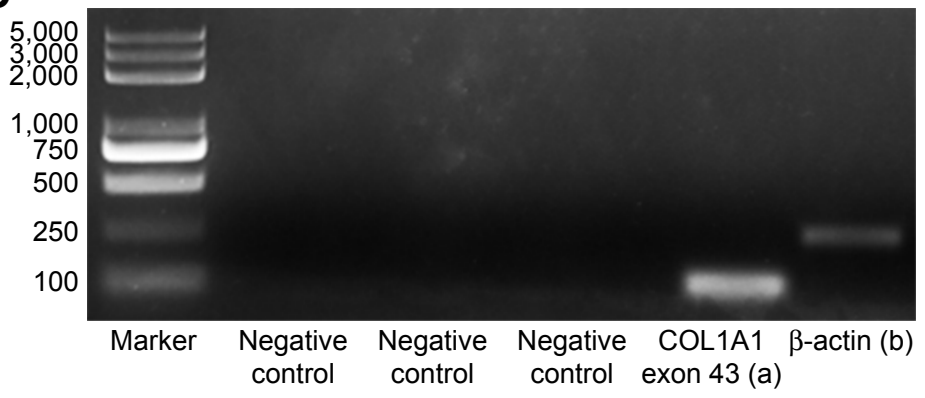

D

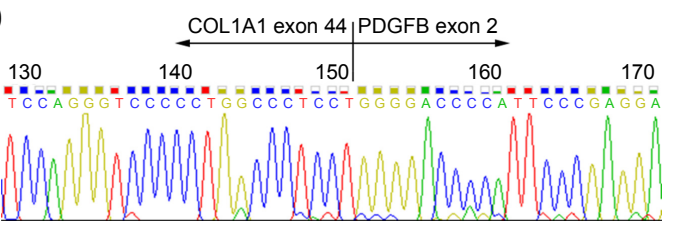

Figure I (A) The PDGFR-beta expression in dermatofibrosarcoma protuberans (DFSP) by immunohistochemical staining in our case (I00 $\times$ and $400 \times$ original). PDGFR-beta is diffuse and strong staining in the paraffin-embedded tissue sample from our patient who received the sunitinib treatment. (B) Negative IHC staining of PDGFR-beta in another tissue sample from patient with DFSP. (C) Expression of COLIAI-PDGFB fusion transcripts. Ethidium bromide-stained agarose gel of reverse transcription polymerase chain reaction products from the paraffin-embedded tissue sample. (a) Amplification product of COLIAI exon 43 forward primer and PDGFB exon 2 reverse primer yields a discrete band, indicating a breakpoint located downstream of COLIAI exon 43. (b) The internal control is verified by amplification of $\beta$-actin gene. (D) Nucleotide sequence analysis showing COLIAI-PDGFB fusion transcripts. The panel shows the nucleotide sequence of COLIAI-PDGFB fusion transcripts. The end of exon 44 in the COLIAI gene was fused with the start of exon 2 in the PDGFB gene detected in the tissue sample.

Abbreviations: PDGFR, platelet-derived growth factor receptor; IHC, immunohistochemistry.

A

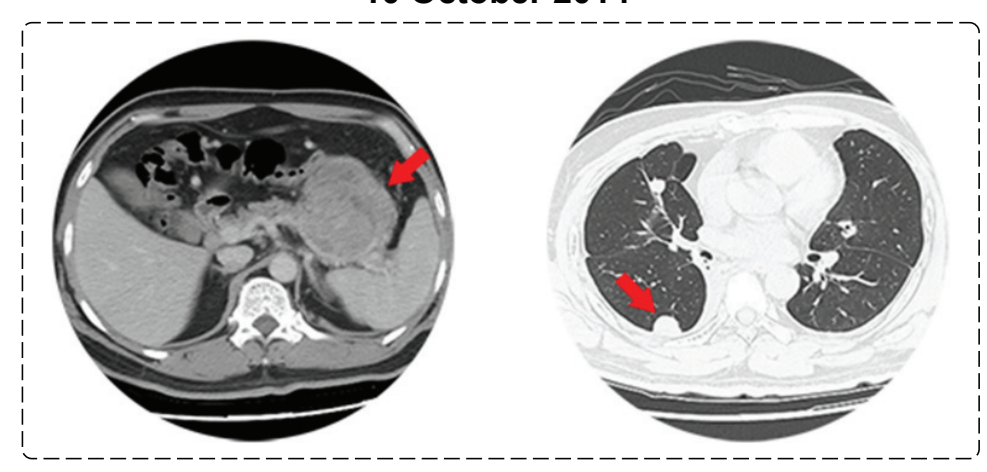

B

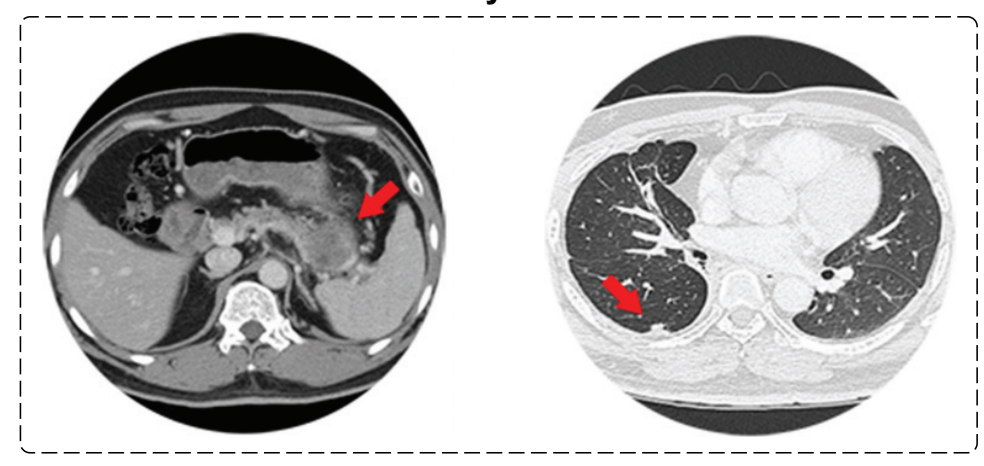

Figure 2 The regular CT scan before and after sunitinib treatment. Radiology of pretreatment (A) and post-treatment (B) findings in our patient under sunitinib treatment. Notes: (A) Baseline with evidence of lung (right red arrow) and pancreas (left red arrow) metastases before starting sunitinib. (B) Evidence of decrease in tumor size on regular scan after 3 months of sunitinib treatment. 
disease and was able to cope with daily activities. A progressive disease was observed by the follow-up investigation in July 2015 (42 months after initial diagnosis). The CT and MRI scans showed an increase in size of the left upper arm mass and metastatic tumor in the lung and pancreas. The patient did not wish to receive any further therapy while we suggested treatment with pazopanib. It resulted in a constant deterioration of the patient's general condition and he died due to multiple organ failure in November 2015 (46 months after initial diagnosis). In total, the progressionfree survival was 9 months in this patient under treatment with sunitinib.

\section{Discussion}

Dermatofibrosarcoma protuberans is known as an uncommon variety of sarcoma, which induces some neoplasms within the deep layers of epidermis. This particular tumor ordinarily spreads gradually and may come to be an elevated nodule. For patients with DFSP, radical surgery could be the only curative procedure. However, this particular tumor has an inclination to relapse following surgical resection. On the other hand, it seldom spreads to other parts of the body.

For the patient in our case report, massive tumor progression was unresponsive to many therapeutic options, including surgery, cytotoxic agents, and microwave ablation. Therefore, due to abundant expression of PDGFR-beta, we initiated imatinib treatment, followed by sunitinib treatment after imatinib failure. During the subsequent 9 months after the sunitinib treatment, the tumor mass on the body surface and both lungs in our patient decreased in size, and he was able to achieve an improvement in quality of life without serious adverse drug reaction. The immunohistochemistry in this patient revealed abundant expression of PDGFR-beta on tumor cells, and the nucleotide sequence analysis revealed COL1A1-PDGFB fusion transcripts. It may be hypothesized that sunitinib may have contributed to an anti-tumor effect to DFSP via PDGFR-dependent mechanisms.

The mechanisms of sunitinib resistance are largely unclear. A study showed that treatment with anti-angiogenic agents would disrupt PDGF signaling between perivascular cells and endothelial cells, and a disturbed balance between destruction of the tumor vasculature and destabilization of the vessel wall could facilitate hematogenous metastasis. ${ }^{8}$ Further studies to interpret the resistance mechanisms of sunitinib on DFSP cells are needed in order to substantiate our clinical observation.

The patient in our case had not responded to conventional chemotherapies including doxorubicin, ifosfamide, dacarbazine, gemcitabine and paclitaxel liposome. The result is concordant with the widely accepted view that traditional cytotoxic agents play a limited role in DFSP. ${ }^{9}$ Previously, researchers have reported promising outcomes in advanced DFSP with sorafenib with 5 months' progression-free survival. It is apparent that tyrosine kinase inhibitors may be effective for patients with advanced DFSP. ${ }^{10}$

In May 2015, it was documented in the scientific literature that sunitinib treatment revealed favorable medical efficacy as another remedy choice for patients with advanced DFSP after imatinib failure. ${ }^{7}$ However, they have not analyzed the relationship between molecular mechanisms and clinical outcomes, which is part of our case report. We treated an advanced DFSP patient with sunitinib after various conventional therapy failures in October 2014 and the patient received a favorable 9-month progression-free survival. In our study, a tentative approach of sunitinib for advanced DFSP would provide helpful information to clinicians.

In our case, the patient with advanced DFSP experienced a favorable outcome in 9-month progression-free survival and a significant improvement of quality of life without serious side effects after sunitinib treatment. The detection of COL1A1-PDGFB fusion transcripts in the samples of this patient is in accordance with one of the drug targets of sunitinib-PDGFR-beta. According to our single-patient experience, sunitinib could represent a tentative treatment for patients with advanced DFSP.

\section{Consent}

Written informed consent was obtained from the patient for publication of this case report and any accompanying images. Consent was obtained using the standardized informed consent forms of the participating institutions. The project and consent process were approved by the ethics board of the Sun Yat-sen University Cancer Center, Guangzhou.

\section{Acknowledgment}

This work was supported by the National Scientific Foundation of China (No 81372887, 81772863).

\section{Disclosure}

The authors report no conflicts of interest in this work.

\section{References}

1. Gloster HM Jr. Dermatofibrosarcoma protuberans. J Am Acad Dermatol. 1996;35(3 Pt 1):355-374; quiz 375-356.

2. Mendenhall WM, Zlotecki RA, Scarborough MT. Dermatofibrosarcoma protuberans. Cancer. 2004;101(11):2503-2508. 
3. Simon MP, Pedeutour F, Sirvent N, et al. Deregulation of the plateletderived growth factor B-chain gene via fusion with collagen gene COL1A1 in dermatofibrosarcoma protuberans and giant-cell fibroblastoma. Nature Genet. 1997;15(1):95-98.

4. McArthur G. Molecularly targeted treatment for dermatofibrosarcoma protuberans. Sem Oncol. 2004;31(2 Suppl. 6):30-36.

5. Bilbao-Meseguer I, Jose BS, Lopez-Gimenez LR, et al. Drug interactions with sunitinib. J Oncol Pharm Pract. 2015;21(1):52-66.

6. Patel KU, Szabo SS, Hernandez VS, et al. Dermatofibrosarcoma protuberans COL1A1-PDGFB fusion is identified in virtually all dermatofibrosarcoma protuberans cases when investigated by newly developed multiplex reverse transcription polymerase chain reaction and fluorescence in situ hybridization assays. Human Pathol. 2008;39(2):184-193.
7. Fu Y, Kang H, Zhao H, et al. Sunitinib for patients with locally advanced or distantly metastatic dermatofibrosarcoma protuberans but resistant to imatinib. Int J Clin Experim Medi. 2015;8(5):8288-8294.

8. Xian X, Håkansson J, Ståhlberg A, et al. Pericytes limit tumor cell metastasis. J Clin Invest. 2006;116(3):642-651.

9. Lemm D, Mügge LO, Mentzel T, Höffken K. Current treatment options in dermatofibrosarcoma protuberans. J Cancer Res Clin Oncol. 2009; 135(5):653-665.

10. Kamar FG, Kairouz VF, Sabri AN. Dermatofibrosarcoma protuberans (DFSP) successfully treated with sorafenib: case report. Clin Sarcoma Res. 2013;3(1):5.

\section{Publish your work in this journal}

OncoTargets and Therapy is an international, peer-reviewed, open access journal focusing on the pathological basis of all cancers, potential targets for therapy and treatment protocols employed to improve the management of cancer patients. The journal also focuses on the impact of management programs and new therapeutic agents and protocols on

\section{Dovepress}

patient perspectives such as quality of life, adherence and satisfaction. The manuscript management system is completely online and includes a very quick and fair peer-review system, which is all easy to use. Visit http://www.dovepress.com/testimonials.php to read real quotes from published authors.

Submit your manuscript here: http://www.dovepress.com/oncotargets-and-therapy-journal 